\title{
La desconsideración de los animales no humanos en la bioética *
}

\section{The disregard for nonhuman animals in bioethics}

\author{
ÓSCAR HORTA
}

\author{
Fundación Española para la Ciencia y la Tecnología/Rutgers University
}

Resumen. Este trabajo defiende dos tesis. En primer lugar, sostiene que, a pesar del amplio uso de animales no humanos en la investigación, esta cuestión no ha sido tomada realmente en serio en el ámbito de la bioética. Esta afirmación es respaldada mediante un rastreo por la literatura reciente. La segunda tesis consiste en que tal desatención no está justificada. Se asume habitualmente que los intereses de los animales no humanos no son moralmente significativos, o al menos que no lo son como los de los seres humanos. Sin embargo, ninguno de los argumentos que defienden esta idea tiene éxito. Los estudios actuales en bioética manifiestan así lo que podemos llamar un prejuicio especista. Más aún, esto conlleva asimismo que el propio uso de animales no humanos como recursos resulta cuestionable.

Palabras clave: antropocentrismo, bioética, especismo, experimentación con animales.

\section{La desconsideración de los animales no humanos en la bioética}

La ética de la investigación con seres humanos ha sido considerada un campo fundamental para la reflexión en bioética. ${ }^{1}$ Ahora bien, en el ámbito de la investigación no sólo se recurre a la participación de seres humanos. Los animales no
Abstract. This paper defends two claims. The first is that, despite the extensive use of nonhuman animals in research, this issue has not been really taken seriously in the field of bioethics. This claim is supported by a survey of recent literature in bioethics. The second claim is that this lack of attention is unwarranted. It is commonly assumed that the interests of nonhuman animals are not morally significant, or at least not as significant as those of humans. However, none of the arguments that have been advanced in defense of this view succeeds. Thus, current studies in bioethics display what may be described as a speciesist bias. Moreover, this also means that the very use of nonhuman animals as resources is questionable.

Key words: anthropocentrism, bioethics, speciesism, animal experimentation.

humanos son utilizados de forma generalizada e institucionalizada en esta en procedimientos de lo más variado. Sin embargo, y en contraste con lo que ocurre en el caso del empleo de sujetos humanos, la atención que ello ha recibido en el campo de la bioética ha sido exigua. $\mathrm{Pa}$ rece asumirse que se trata de una práctica que no suscita ningún problema moral (de hecho, ni siquiera es tratada para in- 
tentar defender su justificación: esta parece darse por obvia).

Hay ciertamente, algunas excepciones a tal postura, y, de hecho, a lo largo de las últimas tres décadas, la cuestión de la consideración moral de los animales no humanos ha sido objeto de una atención creciente. Con todo, la actitud más extendida sigue siendo la que niega al tema la entidad suficiente como para ser discutido con seriedad y detenimiento como una cuestión de primera índole.

El presente artículo tiene dos objetivos a propósito de esto. En primer lugar, defender que, efectivamente, estamos ante una desatención de este tema por parte de la bioética. Esto se llevará a cabo en el apartado 2, que expondrá los resultados de un rastreo bibliográfico acerca de la atención dedicada a la cuestión de la consideración moral de los animales no humanos en revistas de bioética del mundo de habla hispana. El resto del artículo tendrá como finalidad defender que tal desatención no se encuentra justificada. El apartado 3 planteará las distintas condiciones que habrían de darse para que tal justificación efectivamente se diese. Cada una de ellas será explorada en los puntos que sigan a éste. El apartado 4 examinará si la experimentación con animales es numéricamente marginal. El apartado 5 considerará si tenemos motivos para entender que la experimentación con animales no humanos no daña a éstos. El apartado 6 evaluará si hay motivos para considerar que tal daño, en el supuesto de que se dé, restulta mínimo en comparación con aquél que son susceptibles de padecer los seres humanos. El apartado 7 considerará si, al margen de lo indicado en el punto anterior, hay razones normativas por las que el uso de animales no humanos no resulta moralmente problemático. El apartado 8 irá más allá, planteando si hay razones que justifiquen el antropocentrismo moral. Finalmente, el apartado 9 indicará las conclusiones que quepa inferir de todo lo anterior.

He de hacer un apunte antes de entrar en materia. El objeto más básico del presente texto es bastante modesto. Como he dicho, se limita a la consideración de si, a la luz de las evidencias y argumentos disponibles, la experimentación con animales es verdaderamente un tema desatendido y, si, de ser así, ello está justificado o no. Ahora bien, ello no quita que, a partir de los argumentos que aquí se planteen, se puedan inferir conclusiones más sustantivas, relativas no ya a la reflexión sobre la investigación con animales no humanos, sino a esta práctica como tal. Cabe cuestionarse si esta se encuentra justificada. De hecho, aunque por motivos de espacio aquí no profundizaré en esto, tal análisis puede extenderse al uso de animales no humanos como recursos en general, llevando a su cuestionamiento en profundidad.

\section{Un rastreo en la literatura sobre la cuestión}

He indicado que la cuestión de la experimentación con animales no humanos, y en general la de la consideración moral de éstos, no ha recibido hasta ahora más que una atención marginal, si bien esto está comenzando a cambiar. La veracidad de esta afirmación puede comprobarse examinando la atención que recibe la cuestión en la actualidad en la literatura especializada en el campo de la bioética. Por supuesto, un examen exhaustivo de esta resultaría difícilmente abarcable. Pero podemos tomar una referencia algo más modesta pero que resulte, con todo, suficientemente representativa. Aquí voy a considerar, en concreto, la atención prestada en las revistas del campo en el mundo de habla hispana a este tema. He efectuado un rastreo de los artículos publicados en éstas en cinco años recientes (de 2004 a 2008, ambos incluidos), de cara a averiguar 
cuántos se han ocupado de la cuestión que nos ocupa. Lo he hecho distinguiendo aquellos trabajos dedicados al problema concreto de la experimentación con animales no humanos y aquéllos que tratan del tema, más amplio, de su consideración moral en general. He considerado las siguiente doce publicaciones: Acta Bioethica, Cuadernos de Bioética (Argentina),
Cuadernos de Bioética (España), Eidon, ${ }^{2}$ Investigación Clínica y Bioética, Medicina y Etica, ${ }^{3}$ Persona y Bioética, Revista Colombiana de Bioética, Revista de Bioética y Derecho, Revista de la Sociedad Internacional de Bioética, Revista Latinoamericana de Bioética y Vida y Ética. Los resultados fueron los que se exponen a continuación:

Número de artículos tratando (i) la cuestión de la experimentación con animales no humanos y (ii) su consideración moral en general por año y revista analizada

\begin{tabular}{|c|c|c|c|c|c|c|}
\hline $\begin{array}{c}\text { Artículos centrados } \\
\text { en la experimentación / artículos } \\
\text { tratando la consideración moral } \\
\text { en general }\end{array}$ & 2004 & 2005 & 2006 & 2007 & 2008 & Total \\
\hline Eidon & - & - & - & - & - & - \\
\hline Investigación Clínica y Bioética & - & - & - & - & - & - \\
\hline Medicina y Ética & - & - & - & - & - & - \\
\hline Persona y Bioética & - & - & - & - & - & - \\
\hline Revista de la Sociedad & & & & & & \\
\hline Internacional de Bioética & - & - & - & - & - & - \\
\hline Vida y Ética & - & - & - & - & - & - \\
\hline Cuadernos de Bioética (Argentina) & - & - & - & - & - & - \\
\hline Cuadernos de Bioética (España) & - & $1 / 1$ & - & - & - & $1 / 1$ \\
\hline Revista Colombiana de Bioética & - & - & $1 / 1$ & - & - & $1 / 1$ \\
\hline Revista Latinoamericana de & & & & & & \\
\hline Bioética & - & - & - & $-/ 1$ & - & $-/ 1$ \\
\hline Acta Bioethica & - & - & - & $6 / 7$ & - & $6 / 7$ \\
\hline Revista de Bioética y Derecho & - & $-/ 1$ & $-/ 3$ & -13 & $-/ 3$ & $-/ 10$ \\
\hline Total & - & $1 / 2$ & $1 / 4$ & $6 / 11$ & $-/ 3$ & $8 / 20$ \\
\hline
\end{tabular}

La mayoría de las revistas (siete de ellas) no han publicado ningún trabajo acerca de la cuestión. Este es el caso de la Revista de la Sociedad Internacional de Bioética, de la revista colombiana Persona y Bioética, de las españolas Investigación Clínica y Bioética y Eidon, de los Cuadernos de Bioética argentinos, de la también argentina, Vida y Ética y de la mexicana Medicina y Ética. A su vez, tres revistas publicaron un solo artículo sobre el tema: la Revista Colombiana de Bioética (que comenzó su publicación en 2006), ${ }^{4}$ la también colombiana Revista Latinoamericana de Bioética, ${ }^{5}$ y los Cuadernos de Bioética de España. ${ }^{6}$

Sólo dos revistas de las consultadas brindaron un cierto espacio a la cuestión. Una de ellas, la chilena Acta Bioethica dedicó en 2007 un número monográfico a la cuestión, en el que fueron incluidos siete artículos. ${ }^{7}$ La otra, la Revista de Bioética y Derecho, publicada en el estado español, constituye un caso aparte. Esta incluyó en 
el período aquí examinado un total de diez textos tocando la cuestión de la consideración moral de los animales, esto es, la mitad del total publicado ${ }^{8}$ (ninguno de estos trabajos se ha centrado propiamente en la experimentación con animales, aunque en varios casos se refieren a esta de forma más o menos indirecta). ${ }^{9}$ De hecho, esta revista contiene una sección periódica dedicada a este tema, bajo el epígrafe de «Bioética Animal». 10

Cabe destacar dos cuestiones más aparte de lo dicho acerca de los contenidos de las revistas. En primer lugar, es llamativo el hecho de que se han publicado varios artículos que, pese a tratar aspectos muy relacionados con la experimentación o la consideración de los animales no humanos, no prestan propiamente atención a estos temas, pues, o no los tocan en absoluto, o no lo hacen más que de forma totalmente tangencial o marginal. ${ }^{11}$ En segundo lugar, si obviamos el caso de la Revista de Bioética y Derecho, encontramos que la mayoría de los artículos que tocan el tema dan por sentada la justificación de la experimentación con animales no humanos, y/o argumentan básicamente en contra de la consideración moral de estos. ${ }^{12}$

Estos resultados suscriben lo sugerido arriba. Los veinte artículos publicados que se ocupan de temas relativos a los animales no humanos constituyen una parte muy poco relevante del total de artículos publicados por todas estas revistas en cinco años. Si bien puede decirse que en la actualidad el tema de la consideración moral de los animales ha comenzado a ser tomado en serio por una parte de quienes investigan en este ámbito, esta posición es todavía minoritaria. Es claro, pues, el contraste con los vivos debates dados en torno a aquellas cuestiones que involucran a seres humanos.

Hay que indicar finalmente que, a pesar de que este examen se limite a las re- vistas del campo publicadas en lengua castellana, hay motivos para pensar que las conclusiones que cabría desprender de un estudio que incluyese publicaciones de otros contextos lingüísticos no vendrían a diferir en lo esencial de las que he puesto aquí de manifiesto. Por cuestiones de espacio me he limitado a las del contexto lingüístico de esta revista, y para probar esta tesis no llevaré a cabo un recorrido por las revistas más significativas del género en otros idiomas. Pero pondré solamente algún ejemplo al respecto. En el período indicado, ni en la revista internacional Bioethics ${ }^{13}$ ni en su revista hermana Developing World Bioethics se ha incluido ninguna contribución acerca del tema. Y, en otro contexto lingüístico, la revista brasileña Bioética \& Ética Médica no ha publicado tampoco ningún artículo relacionado con la cuestión.

\section{3. ¿Cómo podría estar justificada esta desatención?}

Puede ser que tal desconsideración, pese a resultar tan notable, esté, de todos modos, justificada. Para que ello fuese así tendría que darse al menos una de las siguientes circunstancias:

(A) La magnitud de la utilización de los animales no humanos en el ámbito biomédico ha de ser insignificante en comparación con los casos que afectan a seres humanos.

(B) La utilización de los animales no humanos en el ámbito biomédico ha de ser moralmente irrelevante independientemente de la magnitud que pueda alcanzar.

Esto último podrá ser el caso, a su vez, si se cumple al menos alguna de las dos condiciones siguientes:

(B1) La utilización de los animales no humanos en el ámbito biomédico no implica ningún daño para éstos. 
(B2) El daño sufrido por los animales no humanos en el ámbito biomédico no tiene una relevancia moral mínimamente notable.

A continuación examinaré si efectivamente tenemos motivos para sostener alguna de estas afirmaciones. Será suficiente con que una sola de ellas sea cierta para que debamos considerar que no estamos ante un problema merecedor de atención.

En realidad, cabe apuntar aquí que, al menos desde determinados planteamientos teóricos, un modo de plantear la cuestión como este puede ser que conceda demasiado a quienes acepten la actual desconsideración de la cuestión. Esto podría sostenerse en la medida en la que se defienda que tal desatención viene a mostrar ya de partida una clara falta de sensibilidad, lo cual desde ciertas perspectivas (como, por ejemplo, las de una ética del cuidado o de la virtud), podría ser ya directamente objeto de censura. En cualquier caso, aquí procederé obviando este punto de vista - aun y cuando puede que, al menos en este punto, no esté descaminado-. Pasaré a examinar, pues, si alguna de las condiciones arriba indicadas se cumple.

\section{La dimensión cuantitativa}

El requerimiento que resulta más fácil someter a examen de los arriba apuntados es posiblemente el primero, el relativo a la magnitud de la experimentación con animales no humanos. El número de animales usados con tal fin es muy difícil de calcular con precisión, pero no tanto como para que quede fuera de controversia que resulta enormemente elevado. $\mathrm{Si}$ consideramos, por ejemplo, el contexto europeo, encontramos que según los datos oficiales disponibles, durante 2005 fueron utilizados más de 12,1 millones de animales no humanos en procedimientos de este tipo en la Unión Europea. ${ }^{14}$ Esta cifra resulta, con todo, muy inexacta, pues las regulaciones relativas a los animales usados con tal fin varían mucho de Estado a Estado, y así lo hacen también los datos disponibles y su grado de precisión. ${ }^{15}$ Por otra parte, en muchos otros lugares no hay disponibles datos oficiales sobre esto. En muchos países no existe la obligación de registrar el número de animales empleados, o esta no cubre a todos los que son utilizados. Así, por ejemplo, en Estados Unidos se da la paradoja de que los animales que son empleados de manera más mayoritaria (como son los ratones, ratas, aves, peces y otros animales de sangre fría) no se encuentran incluidos en las cifras oficiales. Esto da como resultado unas cifras muy bajas, que superan apenas el millón de animales por año, ${ }^{16}$ pero que no tienen validez si queremos considerar la experimentación con animales en conjunto (se ha estimado que en realidad el número total de animales podría ascender a unos 18-22 millones). ${ }^{17}$ En otros países, como por ejemplo Rusia, China, India o Brasil, no existen datos oficiales. En algunos, como Japón, se dispone únicamente de cifras obtenidas mediante encuestas a laboratorios e investigadores entre quienes han empleado animales para este fin, que pueden, por supuesto, negarse a dar tal información, o, de proporcionarla, declarar cifras menores de las reales. ${ }^{18}$ Aun así, es posible llevar a cabo algunas extrapolaciones que nos permita estimar cuáles podrían ser las cifras reales a nivel global. En esta línea, en los que posiblemente sean los estudios más rigurosos sobre la cuestión llevados a cabo recientemente, Katy Taylor, Nicky Gordon, Gill Langley y Wendy Higgins han estimado, mediante un modelo estadístico aplicado, que la cifra de animales no humanos utilizados anualmente en los laboratorios ascendería a más de 115 millones; ${ }^{19}$ 
mientras que Andrew Knight, ha elevado tal estimación a unos 127 millones de animales. ${ }^{20}$ De este modo, si consideramos varias décadas podríamos estar hablando de miles de millones de animales. 21

Estamos, pues, ante cifras muy altas en términos absolutos. Pero también lo son en sentido comparativo, como se aprecia si las contrastamos con las que habríamos de manejar en el caso de alguna de las cuestiones que más atención suscitan en el ámbito de la bioética. Por ejemplo, y por tomar uno de los temas que más controversia suscitan, se estima que el número de abortos llevados a cabo a nivel mundial en 2003 fue de 42 millones. ${ }^{22}$ No podemos decir, pues, que la experimentación con animales no humanos resulte un fenómeno reducido, ni que sea marginal en comparación con otros que ocupan a la bioética. La condición (A), en definitiva, no se cumple.

\section{5. ¿Es la experimentación con animales no humanos inocua para estos?}

Nos queda, pues, la condición (B). Un desequilibrio como el arriba apuntado entre la dimensión cuantitativa del fenómeno y la escasa atención que suscita sólo resulta explicable debido a la existencia de un abismo entre el modo en el que se consideran los intereses de los seres humanos y los de los animales no humanos. Se hace preciso, pues, someter a escrutinio los argumentos disponibles a la hora de justificar una diferencia de trato tan notable. Es necesario examinar si los seres humanos poseen intereses moralmente significativos y los animales no humanos no.

Lo primero que esto implica analizar es si se da la subcondición (B1), esto es, si el uso en laboratorios de animales no humanos supone o no un daño para ellos. A este respecto hay que partir del hecho de que toda una serie de procedimientos de este tipo son claramente invasivos. ${ }^{23}$ De manera que habremos de concluir que los animales utilizados en ellos serán dañados si y sólo si poseen la capacidad de sufrir y disfrutar. (B1) se vendrá abajo si concluimos, pues, que efectivamente este es el caso. Para muchos estará fuera de duda que esto es así. El sentido común claramente suscribe esta idea. Sin embargo, no han faltado quienes la han negado. ${ }^{24} \mathrm{Y}$ en la actualidad, teóricos como Peter Harrison y Peter Carruthers continúan haciéndolo. ${ }^{25}$ Hay razones de peso, no obstante, para rechazar tal punto de vista. Disponemos de tres criterios para concluir que la capacidad de sufrir y disfrutar es compartida por al menos un gran número de animales no humanos: los relativos a la conducta, a la lógica evolutiva y a la fisiología:

(a) Conducta. Toda una serie de animales no humanos tienen una conducta plástica cuya explicación más razonable parece pasar por asumir que son conscientes. En lo tocante a experiencias negativas, muchos reaccionan con gesticulaciones, vocalizaciones y movimientos repentinos al sufrir lesiones en sus tejidos semejantes a las que efectúan los seres humanos. ${ }^{26}$ Sin razones sólidas en sentido contrario, asumir que tales comportamientos se dan en presencia de sufrimiento en ciertos casos y como reacciones mecánicas en otros es, como mínimo, antieconómico. Lo mismo sucede en el caso de conductas que sugieren la presencia de experiencias placenteras.

(b) Lógica evolutiva. La posesión de consciencia, y, con ella, de experiencias positivas y negativas, es una herramienta para el control de la homeostasis de los organismos con la capacidad de efectuar movimientos. ${ }^{27}$ Permite a éstos alejarse de aquello que puede afectarles negativamente, y acercarse a lo que puede facilitar su conservación. Sería evolu- 
tivamente paradójico, pues, que quienes no tienen la capacidad de llevar a cabo esto último (como los vegetales ${ }^{28}$ y los hongos) fuesen conscientes. En cambio, parece más que probable que este sea el caso de aquellos seres con capacidades plásticas notables en tal sentido, como sucede en el caso de un gran número de animales. Ahora bien, esta explicación es difícilmente compatible con la idea de que la consciencia no surge hasta la aparición de los seres humanos.

(c) Fisiología. Sabemos que experimentamos dolor y disfrute debido a que tenemos un sistema nervioso centralizado, si bien no sabemos cuál es realmente el proceso mediante el que esto sucede. Estamos muy lejos de llegar a saber qué clase de proceso físico puede dar lugar al surgimiento de la consciencia. ${ }^{29}$ Ello supone que no conocemos el grado de complejidad que un sistema nervioso ha de tener para que pueda surgir un ser consciente. Esto lleva a que a día de hoy no sea posible descartar que haya sistemas nerviosos centrales muy sencillos que puedan ser suficientemente causales para el surgimiento de experiencias. De este modo, no se puede probar que los únicos seres que puedan tener experiencias positivas y negativas sean los humanos. Además, hay otros factores a tener en cuenta, como el hecho de que los organismos de muchos animales estén dotados de nociceptores, o segreguen sustancias que en nuestro caso sirven para paliar el dolor. ${ }^{30}$ Considerando esto conjuntamente con la plasticidad de su conducta y la lógica evolutiva, tenemos fuertes razones para considerar que los vertebrados y un gran número de invertebrados son conscientes.

Todo esto lleva a ver como prácticamente implausible la idea de que sólo los humanos puedan poseer experiencias. Habrá determinadas experiencias que solamente los seres humanos puedan tener, pero ello no implica que sólo ellos tengan experiencias en general. De hecho, también habrá experiencias que los humanos no puedan tener (como por ejemplo, la de la ecolocación). Ciertamente, no está claro dónde hay que situar la frontera que separa a los seres dotados de la capacidad de poseer experiencias negativas y positivas de los que no la poseen. Animales como los poríferos, sin sistema nervioso, o como los equinodermos o los celentéreos, cuyo sistema nervioso no se encuentra centralizado, carecen de ésta. Pero, ciertamente, éste no es el caso de los vertebrados y de un gran número de invertebrados. De este modo, las evidencias a nuestra disposición indican que (B1) se viene abajo.

\section{Sufrimiento físico y psicológico}

Comúnmente se asume, incluso por quienes aceptan que los animales no humanos son susceptibles de sufrir y disfrutar, que su capacidad de hacerlo es radicalmente inferior a la de los seres humanos. ${ }^{31} \mathrm{Po}$ dría pensarse que esto nos debería llevar a modificar las consecuencias de lo concluido en el apartado anterior. Sin embargo, no es así. Desde el momento en que los animales no humanos pueden sufrir y disfrutar, la subcondición (B1) deja de cumplirse. Ello sería así incluso aunque la diferencia apuntada entre la capacidad de sufrir de animales no humanos y humanos fuese cierta. Volveré más adelante sobre esto. Por lo de ahora, cabe indicar que, en cualquier caso, hay razones para rechazar el supuesto en el que descansa este nuevo argumento. La asunción de que las capacidades mentales de los seres humanos vuelven su sufrimiento cualitativamente superior al de otros animales es cuestionable. En primer lugar, hay que indicar que la consideración conjunta de los criterios relativos a la fisiología y a la lógica evolutiva, arriba apuntados, lleva a concluir que no hay motivo para pensar que el dolor experimentado por los seres 
humanos sea de una naturaleza distinta a la del sufrido por los animales no humanos. El sufrimiento que le ocasionan a un conejo las quemaduras oculares provocadas por un químico abrasivo no es menor que el que padecería un ser humano en su misma situación. Ante esto, una respuesta habitual pasa por indicar que, sin embargo, hay sufrimientos y disfrutes de raíz psicológica de los que solo son capaces aquellos seres con ciertas capacidades cognitivas (que, como veremos más adelante, no son todos los seres humanos), y por sostener que estos son incomparablemente superiores a los de los demás animales. ${ }^{32}$ Esta idea es problemática. Puede suceder, sin duda, que ante determinadas situaciones nuestras capacidades cognitivas acrecienten nuestro sufrimiento psicológico — por ejemplo, al hacernos temer lo que sucederá en el futuro si sabemos que nos espera algún mal- . Pero hay otros casos en los que precisamente tales capacidades limitan nuestro sufrimiento, al hacernos comprender, por ejemplo, que una situación de dolor en la que nos encontramos es meramente pasajera. Como ha indicado Bernard Rollin sobre esto:

[S]i los animales están en verdad... inexorablemente aprisionados en lo que está ocurriendo en el aquí y ahora, estamos todavía más obligados a intentar aliviar su sufrimiento, puesto que ellos no pueden aguardar o anticipar su cese, o incluso recordar, por levemente que sea, su ausencia. Si están sufriendo dolor, todo su universo es dolor; no hay horizonte; son su dolor. Así que, si el argumento es de hecho correcto, entonces la consideración del dolor de los animales resulta terrible, puesto que el oscuro universo de los animales no puede, lógicamente, tolerar ningún atisbo de esperanza entre sus confines. 33

De hecho, puede indicarse que nuestra tendencia a sobreestimar el sufrimiento de quienes poseen capacidades intelectuales semejantes a las nuestras puede bien estar influida por nuestra reducida capacidad imaginativa para ponernos en el lugar de quienes no comparten éstas. ${ }^{34}$

De todas formas, el argumento que acabo de exponer es suficiente pero no necesario para rebatir la idea de que mayores capacidades intelectuales implican la posibilidad de alcanzar mayores niveles de sufrimiento y disfrute. Ello es así porque el hecho de que se tenga una mayor capacidad de disfrute o sufrimiento psicológico no hace que se posea tal capacidad de manera general. Para que fuese así deberíamos demostrar que los disfrutes y sufrimientos de carácter psicológico son radicalmente superiores a los de carácter físico. ${ }^{35} \mathrm{Y}$ esta asunción es difícilmente sostenible. Hay disfrutes físicos que contamos entre aquellos más fundamentales para hacer una vida placentera. Y nuestro dolor físico puede ser más intolerable que nuestro sufrimiento psicológico, tanto si hablamos de dolores agudos como de dolencias crónicas (quienquiera que haya padecido una enfermedad o lesión dolorosa puede dar fe de ello).

Por último, es importante tener en cuenta que si la posesión de ciertas capacidades cognitivas implicase la posibilidad de sufrir y disfrutar en grado mayor, esto no trazaría una diferencia entre humanos y no humanos, pues, como veremos más adelante, hay seres humanos, como los niños, que no poseen tales capacidades. Así, se seguiría que éstos tampoco pueden sufrir como los humanos adultos (lo que parece poco plausible).

\section{7. ¿Es el uso de animales no humanos moralmente irrelevante?}

A la vista de lo dicho arriba, nos queda examinar si la subcondición (B2) se encuentra justificada. Lo que hemos de considerar para comprobar si es así es si hay motivos para no tener en cuenta el daño ocasionado a los animales no humanos aun a pesar de que éste sea notable. 
Hasta aquí, todo el razonamiento llevado a cabo ha consistido en la presentación y discusión teórica de ciertos hechos (relativos ya a la magnitud de la investigación con animales no humanos, ya a su impacto en las reflexiones en bioética, ya a la posesión de la capacidad de sufrir y disfrutar por animales no humanos y humanos). En este punto, entramos en el examen de la dimensión propiamente normativa de la cuestión.

Podría pensarse que un primer modo en el que la desconsideración de la experimentación con animales no humanos sería defendible partiría de la asunción de que, aunque los animales son dañados al ser utilizados en los laboratorios, tal daño es insignificante en comparación con los beneficios que los seres humanos pueden obtener de ello. Tal vez podríamos considerar que el modo de examinar si esto es o no efectivamente así pasaría por ponderar conjuntamente el número de los animales no humanos utilizados en este tipo de investigaciones (echando mano de cifras como las arriba indicadas), así como el de los seres humanos que se estima que podrían ser potencialmente beneficiados, $y$, junto a ello, la naturaleza de los daños y beneficios posibles. De tal forma, habría que mostrar que efectivamente las ganancias superan a los costes. Hay varios motivos, sin embargo, por los que esta respuesta no probaría el cumplimiento de (B2). Los expondré a continuación ordenados en función de su relevancia para la cuestión (de menor a mayor):

(ii) Una ponderación cuestionable. En primer lugar, cabe apuntar que una gran parte de la investigación con animales no tiene fines biomédicos, y dentro de ésta otro número muy considerable de procedimientos dista mucho de tener objetivos vitales o siquiera notablemente considerables.

(ii) Compromiso con el agregacionismo. Muchos y muchas de quienes tra- bajan actualmente en bioética no aceptan un punto de vista agregacionista de este tipo (característico, aunque no exclusivo, del utilitarismo). Éste es el caso, por ejemplo, de quienes abrazan posiciones deontológicas o consecuencialistas igualitaristas. Esto les bloquea el acceso a un argumento de este tipo.

(iii) La desconsideración no se sigue. En segundo lugar, aun en el caso de que todos aceptásemos un procedimiento agregacionista, resulta claro que el argumento al que me he referido no mostraría que los intereses de los animales son irrelevantes, sino únicamente que su frustración, de igual modo que la de los intereses humanos, estaría justificada en ciertas condiciones. Así, no proporcionaría propiamente una justificación a la actual desconsideración de la cuestión. Lo que es más, se contrapondría a ésta, puesto que el argumento, para hacerse valer, tendría que plantearse y discutirse, y es precisamente la ausencia de tal discusión lo que aquí lleva a que se plantee esta cuestión. A la luz de esto, podemos retomar una cuestión que había quedado pendiente más arriba: la relativa al supuesto de que el peso de los intereses de un ser viene determinado por sus capacidades intelectuales. He indicado que hay razones para dudar de que esta idea sea correcta. En cualquier caso, el hecho es que, incluso aunque lo fuese, ello no implicaría la pertinencia de la desatención de la cuestión. Lo único que supondría es que, a la hora de examinar ésta podríamos tal vez (en función de la teoría normativa abrazada) tener razones para encontrar justificable el uso de quienes no posean tales capacidades.

(iv) El antropocentrismo no se sigue. El problema fundamental ante el que se encuentra este argumento radica en el hecho de que no es aplicable sólo en el caso de los animales no humanos, sino también en el de los seres humanos. Y, 
como he apuntado con anterioridad, la investigación con sujetos humanos sí es objeto de examen, y sí se considera moralmente problemática. De esta forma, para comprobar si (B2) se cumple, la cuestión a considerar no es si la experimentación con animales no humanos proporciona más o menos beneficios en relación al coste que tiene para éstos. ${ }^{36}$ Es, por el contrario, por qué se considera justificado utilizar a éstos y no a seres humanos con tal fin. No resulta posible dar una justificación de este trato desigual apelando a una simple diferencia cuantitativa entre el peso de los intereses en juego de los humanos y de los demás animales (que, por otra parte, resulta controvertida). Hay una divergencia radical en la manera en la que se ponderan los intereses de los seres humanos y los de los animales no humanos, que obvia de hecho el peso que puedan tener los intereses en juego. Para comprobar si tal divergencia puede estar justificada habrá que examinar si tenemos razones para tener en cuenta los intereses de los seres humanos de forma cualitativamente distinta. En otras palabras, hemos de comprobar si la consideración desfavorable de aquellos que no pertenecen a la especie humana (posición que podemos llamar antropocentrismo) ${ }^{37}$ está justificada. Este será el objeto de la siguiente sección.

\section{Un examen crítico de las defensas del antropocentrismo moral}

A menudo se asume simplemente que la satisfacción de los intereses de los seres humanos tiene un valor superior al de los animales no humanos, sin considerar necesario aportar justificaciones a mayores que sustenten tal idea. Así lo hacen Cora Diamond, Raimond Gaita o Richard Posner. ${ }^{38} \mathrm{O}$ se defiende tal idea, con Aristóteles $\mathrm{o}$, en la actualidad, con autores como Peter Harrison o James Reichmann, ${ }^{39}$ apelando a criterios cuyo cum- plimiento no es susceptible de ser comprobable (como, por ejemplo la posesión de lo que se da en llamar un «estatuto ontológico» superior, no identificable con ningún atributo o capacidad cuya posesión pueda ser verificable o, aun, falsable). ${ }^{40}$ Estas posiciones proceden simplemente asumiendo ya en sus premisas la conclusión a la que buscan llegar, con lo que no avanzan a la hora de proporcionar un fundamento justificado de ésta. En otros casos, el antropocentrismo moral se defiende invocando a atributos cuya posesión sí es comprobable, como han hecho entre otros William Paton, Luc Ferry o Roger Scruton. ${ }^{41}$ Ello sucede cuando se afirma que quienes no tienen ciertas capacidades (como ciertas facultades intelectuales, el uso de un lenguaje u otras) son susceptibles de sufrir un trato desfavorable, y se asume que sólo los seres humanos poseen estas. En otros casos se argumenta, como William Whewell, Lawrence Becker o Michael Goldman, ${ }^{42}$ que los seres humanos tenemos unas relaciones especiales entre nosotros (por ejemplo, de solidaridad, afecto o poder) que no compartimos con otros animales, y que ello justifica que actuemos en perjuicio de éstos.

Ninguno de tales argumentos puede tener éxito. Para que fuese así, los criterios a los que apelan (sean estos referidos a capacidades o a relaciones) deberían cumplir dos requisitos: el de ser moralmente relevantes y el de ser satisfechos por todos los seres humanos y sólo por ellos. Veremos a continuación algunas razones en función de las cuales hay que concluir que tales requerimientos no se cumplen.

Comencemos por el segundo de ellos. Considérense, en primer lugar, los argumentos que apuntan que solo los seres humanos poseen ciertas capacidades cognitivas. El hecho es que tales capacidades no son poseídas por muchos seres 
humanos, como aqueéllos con diversidad funcional intelectual significativa. ${ }^{43}$ Por lo tanto, quienes defendemos la plena consideración moral de éstos habremos de rechazar necesariamente que la posesión de tales capacidades determine la consideración moral que alguien merezca. Tal idea es incompatible con la consideración de estos seres humanos. Puede pensarse que el número de seres humanos que no posee las citadas facultades sería reducido. Esto es, sin embargo, poco relevante: la cuestión es que efectivamente hay seres humanos que no satisfacen los criterios arriba mencionados, independientemente de cuántos sean en proporción. Por otra parte, al margen de esto, ha de tenerse en cuenta que, en realidad, todos los seres humanos carecen de tales capacidades en algún momento de sus vidas: cuando son niños o niñas de corta edad. Cabría pensar que el argumento no tendría validez en este caso, debido al potencial que los niños tienen de desarrollar las capacidades indicadas (tal y como ha argumentado, entre otros, Ronald Dworkin). ${ }^{44}$ Hay, sin embargo, dos motivos para rechazar que esta respuesta pueda ser válida.

El primero - el más importante-, consiste en que apela a un factor que no es el relevante para la cuestión. Tener el potencial para ser, hacer o poseer $x$ no implica ser, hacer o poseer $x$. Mi potencial de ser ministro o alcalde no me proporciona las prerrogativas ligadas a estos cargos. ${ }^{45}$ Podría ser que el potencial para ser, hacer o poseer $x$ implicase la posesión de cierto atributo $y$ que sí sería relevante, pero entonces sería $y$, no el potencial de $x$, lo que habría que considerar, y eso no es lo que sucede aquí. El segundo motivo radica, a su vez, en lo poco intuitiva que realmente resulta la apelación al potencial cuando comprobamos las consecuencias que se derivan de su aceptación. Si la mera potencialidad es lo que determina el interés en vivir de un niño pequeño, o su relevancia normativa, entonces tal interés en vivir será compartido por igual por un óvulo recién fertilizado (y quizás también por un óvulo sin fertilizar, o un espermatozoide). Pero, en cambio, los niños pequeños sin posibilidad de convertirse en adultos no tendrán tal interés, o este carecerá en su caso de relevancia normativa. Si una niña debe ser moralmente considerada sólo por la clase de ser que será en el futuro, una niña con una enfermedad terminal no deberá serlo. Parece, sin embargo, que la niña debe ser respetada por sí misma, por los intereses que posee a día de hoy. ${ }^{46}$

Por otra parte, los criterios apelados en defensa del antropocentrismo también son incumplidos por un gran número de seres humanos cuando se basan en relaciones. Hay muchos que no son objeto de la solidaridad o la simpatía del resto de los seres humanos. Entre ellos cabe contar a niños huérfanos desatendidos o a adultos que viven en soledad, para no hablar de quienes se encuentran prisioneros o viven en situación de esclavitud en distintas partes del mundo. ${ }^{47}$ Estos seres humanos se verán excluidos por quienes defiendan la relevancia para la consideración moral del mantenimiento de tales relaciones.

Todo esto puede tal vez llevarnos, a su vez, a cuestionar la creencia de que tales criterios pueden ser moralmente relevantes, esto es, la primera condición que las defensas del antropocentrismo deberían satisfacer. ${ }^{48}$ En cualquier caso, el incumplimiento de la segunda condición es ya suficiente para tener que abandonar esta posición. ${ }^{49}$

A la luz de esto, se ha de rechazar la idea de que los intereses de los seres humanos cuentan por sí mismos más que los que puedan poseer otros animales aun cuando el peso de estos últimos sea mayor. Tal idea puede ser descrita como una 
forma de especismo, esto es, de consideración desventajosa injustificada de quienes no pertenecen a una cierta especie (o especies). ${ }^{50}$ No hay motivo, pues, para no tener en cuenta plenamente los intereses de los animales no humanos. De este modo, la subcondición (B2) se ve también rebatida. Así, hay que concluir que ninguna de las posibles maneras en las que la no consideración de la cuestión de la investigación con animales no humanos podría estar justificada tiene éxito. Lo que es más: de cumplirse (B2) se seguiría que tampoco la investigación con toda una serie de seres humanos merecería atención.

En realidad, cabe apuntar, el rechazo del especismo antropocéntrico constituye una razón suficiente para refutar (B2). Pero es de interés tener en cuenta que no es, sin embargo, una razón necesaria para ello. El especismo antropocéntrico sostiene que la pertenencia a la especie humana es un criterio moralmente relevante. Pero ni siquiera de esta idea se seguiría necesariamente la de que los de los animales no humanos sean irrelevantes. Esta conclusión sólo puede inferirse si se asume una posición especista más particular: la que mantiene que no existe ningún criterio para la consideración moral que los animales no humanos puedan cumplir. El motivo por el que esto es así es el siguiente. A la luz de lo que hemos visto arriba en lo relativo a los números de animales no humanos utilizados y a su capacidad de sufrir, la desconsideración de los animales no humanos en la bioética estaría justificada únicamente si sus intereses contasen no ya menos que los de los seres humanos, ni siquiera bastante menos, sino prácticamente nada. Más aún, es posible que solo con que contasen algo, por poco que fuese, sería suficiente para tener que ser tenidos en cuenta de forma significativa, lo que no ocurre a día de hoy. El motivo no es difícil de adivi- nar. Si los intereses de los animales no humanos contasen verdaderamente algo, tendría que llegar un momento, si comenzásemos a considerarlos de forma conjunta, en el que su significación moral sobrepasase a la poseída por algunos intereses humanos. Robert Bass ha indicado acerca de esto:

[U]na vez admites que los intereses de los animales tienen algún peso puedes decir, por ejemplo, que una vida humana tiene un valor mil veces mayor que el de la vida de una rata, pero tendrás también que derivar la consecuencia de que no compensa sacrificar 1.001 ratas para salvar una vida humana. ${ }^{51}$

Ahora bien, dado el inmenso número de animales no humanos utilizados en los laboratorios, bien puede decirse que el punto en el que una consideración realmente seria de sus intereses resultaría ineludible tendría que haber llegado en algún momento; de hecho, hace ya bastante tiempo.

\section{Conclusión}

Hemos visto que el número de animales no humanos empleados para la investigación en distintos procedimientos es elevadísimo. Asimismo, hemos comprobado que no tenemos razones para suponer que estos no son dañados al ser objeto de tal uso. Hemos constatado que, sin embargo, los estudios actuales en el ámbito de la bioética no le prestan prácticamente ninguna atención a esta cuestión. Tal desconsideración podría verse justificada si no tuviésemos absolutamente ningún motivo para considerar verdaderamente los intereses de los animales no humanos. Sin embargo, hemos visto también que tenemos razones para entender que este no puede ser realmente el caso. Los argumentos a favor del antropocentrismo moral no resultan convincentes. Así, fracasa la idea de que tal daño pueda resultar moralmente irrelevante. A la vista de esto, procede de- 
cir que la falta de atención que el tema ha tenido hasta el momento en el ámbito de la bioética resulta inexcusable. Podemos considerar, por ello, que los estudios actuales en bioética muestran lo que podemos llamar un prejuicio antropocéntrico, que, a la vista de los argumentos arriba indicados, puede ser tildado de especista. Más aún, en realidad, y aunque ello no haya sido el objeto de este artículo, cabe decir que de tales argumentos se pueden derivar otros corolarios mucho más signi- ficativos. Éstos cuestionan en profundidad no ya el trato que se da actualmente a los animales no humanos, sino su propia utilización como recursos a nuestra disposición. Y lo dicho en el ámbito de la experimentación con animales no humanos puede ser extendido al uso de estos en otros ámbitos, como son el culinario-alimenticio, el de la confección de ropa o el del entretenimiento, pues ninguno de ellos continúa resultando aceptable a la luz de los argumentos aquí considerados.
* Este trabajo ha sido realizado en el marco del proyecto de investigación «Bioethical Underpinnings for the Consideration of Practical Dilemmas concerning the Interest in Living» (exp. 2008-0423) con la financiación del Ministerio de Ciencia e Innovación de España.

1 A este respecto puede considerarse, por supuesto, la Declaración de Helsinki, o los distintos artículos dedicados a la cuestión en la Declaración Universal sobre Bioética y Derechos Humanos de la UNESCO —así como otros textos que han alcanzado una cierta relevancia, como el informe Belmont-.

2 Aunque esta publicación trata también otras cuestiones además de las de propias de la bioética, la atención central que presta a esta disciplina justifica su inclusión aquí.

3 Podríamos pensar que en este caso estamos ante una publicación centrada en la ética médica, pero el hecho es que Ésta no se limita a cuestiones como la práctica clínica, sino que trata también temas como la investigación, de modo que es pertinente incluirla aquí. De hecho, como se apuntará más adelante, esta revista sí publicó un artículo tangencial a la cuestión.

4 A. Mrad de Osorio, «Ética en la investigación con modelos animales experimentales. Alternativas y las 3 Rs de Russel. Una responsabilidad y un compromiso ético que nos compete a todos», Revista Colombiana de Bioética, 1, 2006, 163-83.

5 A. Marcos Martínez, «El "Proyecto Gran Simio" y los fundamentos filosóficos de la biopolítica», $R e$ vista Lationamericana de Bioética, 12, 2007, 60-75.

6 A. Pardo Caballos, «Ética de la experimentación animal: directrices legales y éticas contemporáneas», Cuadernos de Bioética, 16, 2005, 393-417. (Cabe apuntar que esta revista dedicó un número monográfico a la cuestión en 1996).

7 A. R. Concepción Alfonso, R. de la Peña Pino y J. García Capote, «Acercamiento al accionar ético-moral del científico que trabaja con animales de experimentación», Acta Bioethica, 13, 2007, 9-16; J. Fernández Hernández e Y. M. Heuze de Icaza, «El programa interno para el cuidado y uso de los animales de laboratorio en las instituciones biomédicas docentes, de investigación científica e industria farmacéutica», Acta Bioethica, 13, 2007, 17-24; E. Rodríguez Yunta, «Ética de la investigación en modelos animales de enfermedades humanas», Acta Bioethica, 13, 2007, 25-40; M. P. Vinardell Martínez-Hidalgo, «Alternativas a la experimentación animal en Toxicología: situación actual», Acta Bioethica, 13, 2007, 41-52; J. A. Álvarez-Díaz, «La controversia sobre la vivisección», Acta Bioethica, 13, 2007, 53-60; A. Bota Arqué, «Animales transgénicos como organismos artificiales», Acta Bioethica, 13, 2007, 61-70; C. Valdebenito, «Definiendo homo sapiens-sapiens: Aproximación antropológica», Acta Bioethica, 13, 2007, 71-78.

${ }^{8}$ M. Escartín Gual, «AAnimales? "No nada”. Reflexiones de ética animal», Revista de Bioética y Derecho, 4, 2005, 14-21; M. Tafalla, «Sobre perros y justicia: a propósito de la prohibición del sacrificio de perros abandonados en Catalunya», Revista de Bioética y Derecho, 6, 2006, 30-34; O. Campos Serena, «La importancia del sufrimiento», Revista de Bioética y Derecho, 7, 2006, 50-57; P. Casal, «El planeta sin los simios», Revista de Bioética y Derecho, 8, 2006, 21-24; E. López-Almansa Beaus, «Legislación europea sobre protección de los animales tras el Plan de Acción 2006-2010», Revista de Bioética y Derecho, 9, 2007, 24-28; C. Velayos Castelo, «Animales reales en el arte, o sobre los límites éticos de la capacidad creadora», Revista de Bioética y Derecho, 10, 2007 24-38; M. C. Castro Álvarez, «Algunas reflexiones sobre la legislación internacional y española en materia de animales de compañía (con atención especial a la normativa de la Comunidad Autónoma de Cataluña)», Revista de Bioética y Derecho, 11, 2007, 24-33; M. Escartín y T. Ausín, «El mundo académico ante las corridas de 
toros», Revista de Bioética y Derecho, 12, 2008 , 24-32; N. Querol Viñas, "Violencia hacia animales por menores... ¿cosas de niños?», Revista de Bioética y Derecho, 13, 2008, 12-28; T. Giménez-Candela, «New rules to ensure the protection of animals in Spain. Spanish Animal Welfare Act. 32/2007. $7^{\text {th }}$ November (BOE. 268, $8^{\text {th }}$ November 2007. Number 19321)», Revista de Bioética y Derecho, 14, 2008, 25-28. La revista también incluyó otras colaboraciones tocando la cuestión en forma de entrevista o de crónica.

9 Se podría decir esto de los artículos, referidos en la nota anterior, de Elena López-Almansa y Teresa Giménez-Candela, así como en los de Monserrat Escartín, Olga Campos y Paula Casal.

10 Cabe apuntar asimismo que, a mayores de esto, en mayo de 2010 esta revista dedicó un número especial a esta cuestión, con tres trabajos: A. Gonçalves, C. Isaías y $\mathrm{N}$. de Campos «O animal não-humano e seu status moral para a ciência e o Direito no cenário brasileiro», Revista de Bioética y Derecho, 19, 2010, 2-7; D. Favre, «Ethical Duties Based upon Animal Interests», Revista de Bioética y Derecho, 19, 2010, 8-13; F. Leyton, «Literatura básica en torno al especismo y los derechos animales», Revista de Bioética y Derecho, 19, 2010, 14-16.

11 Véase L. Palazzani, «Cuerpo y sujeto en bioética», Cuadernos de Bioética, 15, 2004, 17-27; J. J. García, «La realidad humana como pauta ética en la filosofía de Xavier Zubiri», Cuadernos de Bioética, 16, 2005, 375-392; J. F. Sellés, «Distinción entre el hombre y el animal en sus funciones vegetativa y cinética», Persona y Bioética, 11, 2007, 39-53. G. Hottois, «Panorama critique des éthiques du monde vivant», Revista Colombiana de Bioética, 1, 2006, 7-34; y C. E. Maldonado, «Reflexiones filosóficas sobre la experimentación en seres vivos», Revista Colombiana de Bioética, 1, 2006, 89-103; Véase A. Sutton, «Do Human-Animal Hybrids and Chimeras Mean the Abolition of Man?», Medicina y Ética, 1, 2008, 13-28. Es llamativo que, pese a sus títulos, ninguno de los tres últimos trabajos trate la cuestión. El primero, aunque da algún argumento que tal vez de pasada podría ser tratado en el examen del tema, viene realmente a constituir una crítica a una serie de propuestas en ética medioambiental. El segundo consiste en una reflexión sobre la investigación científica que no entra en cuestiones morales relativas al daño ocasionado a los animales no humanos. El tercero examina la creación de híbridos entre humanos y animales sin tocar la consideración de estos últimos. No he listado aquí otros artículos que tratan cuestiones que implícitamente suponen el problema de la consideración moral de los animales, como la de la «dignidad humana». Sí he incluido el artículo de Juan Fernando Sellés porque, aunque consiste únicamente en una defensa de la idea de que hemos que considerar a los seres humanos y no a los animales no humanos, al menos se acerca al tema, y merece por ello ser apuntado.
$12 \mathrm{La}$ única excepción sería el trabajo de Jorge Alberto Álvarez-Díaz.

13 Esta revista incluyó sólo dos textos tangenciales a la cuestión. Uno de ellos considera la cuestión de las enfermedades de los animales, pero se focaliza en particular en el modo en el que este problema afecta a los seres humanos, más que a los animales no humanos como tales. Véase H. McCallum y B. A. Hocking, «Reflecting on Ethical and Legal Issues in Wildlife Disease», Bioethics, 19, 2005, 336-347. El otro ni siquiera se centra en la cuestión, sino que trata de la experimentación con embriones humanos, resultando relevante para el tema únicamente porque en él se busca defender explícitamente una propuesta que excluya moralmente a los animales no humanos. Véase J. Deckers, "Why Current UK Legislation on Embryo Research Is Immoral: How the Argument from Lack of Qualities and the Argument from Potentiality Have Been Applied and Why They Should Be Rejected», Bioethics, 19, 2005, 251-71.

14 Comisión de las Comunidades Europeas (Unión Europea), Informe de la Comisión al Consejo y al Parlamento Europeo: Quinto informe sobre las estadísticas relativas al número de animales utilizados para experimentación y otros fines cientificos en los estados miembros de la Unión Europea \{SEC(2007) 1455\}, COM(2007) 675, Bruselas, Unión Europea, 2007 , p. 4. Se trata de la suma de las cifras de 2005 de los distintos países de la Unión Europea, a excepción de Francia, en cuyo caso se agregó la cifra de 2004.

15 En el Reino Unido, donde el registro de los procedimientos se rige por criterios menos relajados que los de otros países europeos, las cifras recogidas apuntan a que en los últimos años la cifra media de animales empleados cada año osciló en torno a los 3 millones de animales (3.619.540 en 2009). Home Office, United Kingdom, Statistics of Scientific Procedures on Living Animals, Great Britain, 2009, London, The Stationery Office, 2010, p. 23.

16 La cifra exacta es de 1.131.076 animales. Véase Animal and Plant Health Inspection Service, United States Department of Agriculture, Annual Report Animal Usage by Fiscal Year, 2009, 2010, p. 2.

17 M. Mukerjee, «Trends in animal research», Scientific American, 276, 1997, 86-93. Un repaso de las tendencias en el uso de animales no humanos en este ámbito en Estados Unidos puede verse en A. N. Rowan, y F. M. Loew, «Animal Research: A Review of Developments, 1950-2000», en D. J. Salem y A. N. Rowan (eds.), The State of the Animals 2001, Washington, HSUS, 2001, 111-20.

18 Véase K. Yagami, T. Mashimo, F. Sekiguchi, F. Sugiyama, K. Yamamura y T. Serikawa, «Survey of Live Laboratory Animals Reared in Japan (2009)», Experimental Animals, 59, 2010, 531-35. Según este estudio, en junio de 2009 había 11.337.334 animales 3 en los laboratorios de este país.

19 K. Taylor, N. Gordon, G. Langley y W. Higgins «Estimates for Worldwide Laboratory Animal Use in 
2005», Alternatives to Laboratory Animals, 36, 2008, 327-42. Las autoras de este estudio se basaron en las cifras diponibles para 2005, realizando extrapolaciones estimativas en función de factores como la ratio de producción científica sobre la cuestión. Este método conlleva el problema de que tal ratio es en realidad menor en los países no anglosajones y no occidentales, por ser el idioma de las principales revistas científicas en inglés.

20 A. Knight, «127 million non-human vertebrates used worldwide for scientific purposes in 2005», Alternatives to Laboratory Animals, 36, 2008, 494-96.

${ }^{21}$ Cabe apuntar, por otra parte, el contraste existente entre la ausencia de publicaciones relativas a la experimentación con animales en el caso de la bioética y la multitud de publicaciones científicas en base a éstas. Véase S. Zhao, E. Liu, Y. Chu, H. Zheng, Sh. Kitajima y M. Morimoto, «Numbers of Publications Related to Laboratory Animals», Scandinavian Journal of Laboratory Animal Science, 34, 2007, 81-86.

22 G. Sedgh, S. Henshaw, S. Singh, E. Åhman e I. H. Shah, «Induced Abortion: Rates and Trends Worldwide», Lancet, 370, 2007, 1338-45.

23 Descripciones clásicas de procedimientos en los que son utilizados animales no humanos son R. D. Ryder, Victims of Science. The Use of Animals in Research, London, Davis-Poynter, 1975; J. Vyvyan, In Pity and in Anger: A Study of the Use of Animals in Science, Marblehead, Micah Publishing, 1988; G. Langley (ed.), Animal Experimentation: The Consensus Changes, London, MacMillan, 1990; P. Singer, Liberación animal, Animal Liberation: A New Ethic for Our Treatment of Animals, segunda edición, New York, Random House, 1990, capítulo 2. Se echa en falta una mayor cantidad de trabajo, más actual, sobre la cuestión, si bien la gran mayoría de lo indicado en estos estudios continúa estando plenamente vigente.

24 El locus classicus aquí es, por supuesto, R. Descartes, Discours de la méthode, Paris, Vrin, 1930.

25 Véase P. Harrison, «Do Animals Feel Pain?», Philosophy, 66, 1991, 25-40; P. Carruthers, The Animal Issue: Moral Theory in Practice, Cambridge, CUP, 1992.

26 Trabajos fundamentales en la cuestión son M. S. Dawkins, Through Our Eyes Only? The Search for Animal Consciousness, New York, W. H. Freeman, 1993; D. R. Griffin, Animal Minds, Chicago, University of Chicago Press, 1992.

27 Véase A. R. Damasio, The Feeling of What Happens: Body and Emotion in the Making of Consciousness, San Diego, Harcourt, 1999, pp. 23-25.

28 Por supuesto, también el criterio de la conducta y el de la fisiología niegan la posesión de consciencia a seres vivos no animales. Véase sobre esto Y. Bonnardel, «Quelques réflexions au sujet de la sensibilité que certains attribuent aux plantes", Les cahiers antispécistes, 5, 1992, http://www.cahiers-antispecistes.org/ article.php3?id_article $=40$.
29 De hecho, no faltan quienes, con Colin McGinn, consideran que nunca llegaremos a solucionar este problema. Véase C. McGinn, Consciousness and Its Objects, Oxford, OUP, 2004.

30 Acerca de las distintas evidencias en este sentido véase por ejemplo D. De Grazia, Taking Animals Seriously: Mental Life and Moral Status, Cambridge, CUP, 1996, capítulo 5; L. U. Sneddon, «Evolution of Nociception in Vertebrates: Comparative Analysis of Lower Vertebrates», Brain Research Reviews, 46, 2004, 123-30; J. A. Smith, «A Question of Pain in Invertebrates», ILAR Journal, 33, 1991, http://dels. nas.edu/ilar_n/ilarjournal/33_1_2/V33_1_2Question. shtml.

31 Una idea así es sugerida en R. Gaita, The Philosopher's Dog: Friendships with Animals, London, Routledge, 2003. No se debe confundir esta idea con la de que los intereses de los seres humanos tienen más peso, como se defiende en R. G. Frey, Rights, Killing and Suffering: Moral Vegetarianism and Applied Ethics, Oxford, Basil Blackwell, 1983.

32 Podría pensarse que la (por otra parte muy denostada) distinción milliana entre «placeres superiores» y "placeres inferiores» apuntaría en esta dirección. Véase J. S. Mill, Utilitarianism, en Collected Works, vol. X, London, Routledge and Kegan Paul, 1969, 203-59, pp. 211-12. Con todo, se ha discutido si lo que Mill quería apuntar con tal distinción sería una inconmensurabilidad en el valor de los placeres o, simplemente, que hay placeres que en menor cantidad pueden ser más valiosos que otros en más cuantía. Véase Ch. Schmidt-Petri, «Mill on Quality and Quantity», Philosophical Quarterly, 53, 2003, 102-4.

33 «[I]f animals are indeed... inexorably locked into what is happening in the here and now, we are all the more obliged to try to relieve their suffering, since themselves cannot look forward to or anticipate its cessation, or even remember, however dimly, its absence. If they are in pain, their whole universe is pain; there is no horizon; they are their pain. So, if the argument is indeed correct, then animal pain is terrible to contemplate, for the dark universe of animals logically cannot tolerate any glimmer of hope within its borders» (B. E. Rollin, The Unheeded Cry: Animal Consciousness, Animal Pain and Science, Oxford, OUP, 1989, p. 144). El mismo argumento se defiende en J. J. Thomson, The Realm of Rights, Cambridge, Harvard University Press, 1990, p. 292.

34 Considérese que, aunque muchos podrían preferir ser Sócrates insatisfecho a un cerdo insatisfecho, es también probable que prefiriesen ser Sócrates insatisfecho antes que, por ejemplo, un ser dotado de unas capacidades cognitivas muchísimo mayores que las de Sócrates de naturaleza totalmente diferente a la nuestra (como los que aparecen en ciertos relatos de ciencia ficción). La preferencia por la vida de Sócrates parece debida fundamentalmente a un apego por nuestra clase de vida. 
35 Obsérvese que lo que no procede es asumir que unas mayores capacidades para el sufrimiento psicológico pueden hacer que padezcamos un mayor dolor físico (como se defiende en M. P. T. Leahy, Against Liberation: Putting Animals in Perspective, London, Routledge, 1991).

36 Véase al respecto M. H. Bernstein, «Marginal Cases and Moral Relevance», Journal of Social Philosophy, 33, 2002, 523-39.

37 El término «antropocentrismo» ha sido usado para referir posiciones de lo más diverso, tanto en el campo de la ética como en ámbitos como en el epistemológico. Tales sentidos no se implican unos a otros. Aquí emplearé este término exclusivamente para denotar la posición arriba caracterizada.

38 C. Diamond, The Realistic Spirit: Wittgenstein, Philosophy and the Mind, Cambridge, MIT Press, 1995; R. Posner, «Animal Rights: Legal, Philosophical and Pragmatical Perspectives», en C. Sunstein y M. Nussbaum (eds.), Animal Rights: Current Debates and New Directions, Oxford, OUP, 2004, 51-77.

39 Véase Aristóteles, Política, Madrid, Tecnos, 2004, 1254a 21-1254b 15; J. Reichmann, Evolution, Animal «Rights» and the Environment, Washington, The Catholic University of America Press, 2000; P. Harrison, «Theodicy and Animal Pain», Philosophy, 64, 1989, 79-92.

40 Tales criterios pueden consistir tanto en atributos (como, por ejemplo, un supuesto «estatuto ontológico») como en relaciones (como, por ejemplo, una «unidad de destino común»). Las defensas del antropocentrismo apelando a razones religiosas entrarían en este tipo.

41 W. Paton, Man and Mouse, Oxford, OUP, 1984; L. Ferry, Le nouvel ordre écologique: l'arbre, l'animal et l'homme, Paris, Grasset, 1992; R. Scruton, Animal Rights and Wrongs, London, Metro, 1996.

42 W. Whewell, Lectures on the History of Moral Philosophy in England, London, John Parker, 1852, p. 223; L. Becker, «The Priority of Human Interests», en H. Miller y W. Williams (eds.), Ethics and Animals, Clifton, Humana Press, 1983, 225-42; M. Goldman, «A Trascendental Defense of Speciesim», Journal of Value Inquiry, 33, 2001, 59-69.

43 El término «diversidad funcional» viene precisamente a denotar una actitud contraria a la idea de que la posesión de ciertas capacidades, o más bien posibilidades funcionales, determine un patrón de lo que es el grupo humano, del que caen por debajo algunos individuos. Con todo, ésta no es una expresión del todo adecuada. El término diversidad tiene sentido en la medida en que hay una pluralidad en las posibilidades de distintos individuos. No es un cierto individuo, o una cierta minoría de individuos, pues, quien tiene tal diversidad funcional; esta se predica en conjunto. (Curiosamente, la terminología de las capacidades sí es comúnente mantendida cuando se habla de animales no humanos. Aquí no he cuestionado tal uso para no complicar aun más el análisis, pero parece que éste no debería ser visto como procedente por quienes rechazan tal terminología al hablar de seres humanos).

44 R. Dworkin, Life's Dominion: An Argument about Abortion and Euthanasia, London, Harper Collins, 1993, p. 185.

45 H. T. Engelhardt Jr., The Foundations of Bioethics, Oxford, OUP, 1986, p. 111.

46 Algo que ya se apuntó Leonard Nelson en su momento. Véase L. Nelson, System of Ethics, New Haven, Yale University Press, 1956, p. 143.

47 Con esto vemos que el argumento considerado hasta aquí, el de que los criterios esgrimidos en defensa del antropocentrismo no son cumplidos por todos los seres humanos, opera también cuando estos apelan a relaciones, no sólo cuando se refieren a capacidades. Es necesario corregir en este punto, pues, lo que se asume comúnmente en la literatura sobre la cuestión - véase como ejemplo, D. A. Dombrowski, Babies and Beasts: The Argument from Marginal Cases, Chicago, University of Chicago, 1997-. Por otra parte, hay algo más que decir en lo que atañe a su nomenclatura. Este argumento ha sido conocido de manera común como «argumento de los casos marginales». Empero, tal denominación resulta totalmente inapropiada, al asumir que hay un centro y unos márgenes en lo que atañe a circunstancias definidoras de la pertenencia a una especie, una idea que precisamente este argumento viene a rebatir. No incurre en estos problemas, sin embargo, el término "argumento de la superposición de especies».

48 Hay en realidad otro argumento, más complejo, para rechazar que esto sea así, el que podemos llamar argumento de la relevancia. Éste opera una vez asumimos la premisa de que lo moralmente relevante debe basarse en aquello que es ónticamente relevante. Dado esto, podemos argumentar, en aquellas decisiones en las que lo que está en juego es la posibilidad de dañar o beneficiar a un cierto individuo, el criterio básico para su consideración será precisamente su posibilidad de ser dañado o beneficiado.

49 Podría decirse aquí que habría un único criterio que podríamos decir que todos los seres humanos, y sólo ellos, cumplirían: la posesión de una dotación genética humana. Pero, en realidad, este criterio no vendría a ser más que una formulación de una defensa definicional del antropocentrismo.

50 Para una explicación más detallada de esta definición véase O. Horta, «What Is Speciesism?», Journal of Agricultural and Environmental Ethics, 23, 2010, 243-66.

51 «[O]nce you admit some weight to animal interests, you may say, for example, that a human life is a thousand times as valuable as a rat's life, but you will also have to draw the consequence that it is not worth sacrificing 1001 rats to save a human life» (R. Bass, «Undermining Indirect Duty Theories», Between the Species, 6, 2006, http://cla.calpoly.edu/bts/issue_06/06 bass.pdf, p. 7). 